\title{
The Correlation between Phalangeal Quantitative Ultrasonography and Dual Energy X-ray Absorptiometry in Women with Premature Ovarian Failure
}

\author{
Tandip S Mann*, Alison H McGregor, Rajesh Patel
}

\begin{abstract}
Objectives - With the growing demand for bone densitometry services there is a need for simple, cost-effective and ideally mobile devices which can identify individuals who are at risk of osteoporotic fracture. When new devices are evaluated, it is useful to examine the correlation with the established 'gold standard' technique of dual x-ray absorptiometry (DXA). This study examined the correlation between quantitative ultrasound (QUS) measurements performed at the phalanges and conventional DXA measurements of the spine and hip in women with premature ovarian failure - a known risk factor for osteoporosis. Methods - Thirteen white Caucasian women suffering from premature ovarian failure and 19 age- and sex-matched controls were recruited into the study. DXA measurements were performed at the spine and hip, followed by quantitative ultrasonography at phalanges II-V of the non-dominant hand. Results - Significant correlations were observed between the bone transit time (BTT) value from the Bone Profiler and bone mineral density measured at the spine $(r=0.66)$. The spine $Z$-scores also correlated with many of the ultrasound values $(r=0.44-0.63)$. Significant inverse correlations were observed between BMI, weight and ultrasound parameters $(r=$ 0.48 to $\mathbf{- 0 . 7 8}$ ). Conclusion - We have reported moderate but significant correlations between phalangeal QUS and DXA parameters. The strongest correlation was observed between BTT and spine BMD, as well as between the Z-scores from the two devices. QUS parameters also demonstrated an inverse correlation with weight and BMI.
\end{abstract}

KEYWORDS: Bone mineral density, osteoporosis, premature ovarian failure, DXA, quantitative ultrasound.

\section{INTRODUCTION}

In many developed countries, osteoporosis is now recognised as one of the most serious problems in public health [1-3]. For a 50-year-old white woman, the life-time risk of suffering a fragility fracture is estimated to be $30-40 \%$, which compares with the figures for breast cancer and cardiovascular disease of $9-12 \%$ and $30-40 \%$ respectively [3]. The increased recognition of the impact of osteoporosis on the lives of elderly people and the consequent costs of healthcare

*To whom correspondence should be addressed:

Tandip Mann

Room 7L17, 7th Floor, Lab Block, Faculty of Medicine

Department of Biosurgery and Surgical Technology

St Dunstan's Road,Charing Cross Hospital, London, United Kingdom W6 8RP

Tel: 442083834437

Email: Tandip.mann@imperial.ac.uk has led to the development of a variety of new treatments for preventing fractures [4-7]. Scans to measure bone mineral density (BMD) using the technique of dual x-ray absorptiometry (DXA) are widely believed to be the most effective way of identifying patients at risk of fracture and targeting these treatments appropriately [8,9]. Conventionally, the hip and spine are regarded as the most important measurement sites because fractures at these sites have the greatest impact on quality of life, morbidity and mortality of patients.

An individual's BMD undergoes progressive changes throughout life. There is rapid skeletal growth until peak bone mass (PBM) is reached at around age 30 [10], after which there is minimal change until the menopause in women. Following menopause, there is an approximately linear loss of BMD with increasing 
age [11]. PBM occurs in the third decade of life, but an inadequate nutritional intake of calcium [12] can prevent optimum peak bone mass being achieved. Low levels of physical activity during puberty can also lend to suboptimal bone density in later life [13]. Certain medical conditions and therapies can also affect bone metabolism and thus adversely affect BMD [14]. Longterm corticosteroid use is one such example, causing changes to trabecular bone structure, as well as reducing BMD [15]. Other factors known to compromise BMD include a previous atraumatic fracture or maternal hip fracture, a previous report of X-ray osteopenia, a positive family history of osteoporosis [16], a body mass index (BMI) of less than $19 \mathrm{~kg} / \mathrm{m} 2$, smoking and suffering from rheumatoid arthritis [17]. Sex hormone deficiency, most notably oestrogen in females, is a well established contributor to the pathogenesis of osteoporosis [18]. Menopause results in oestrogen deficiency, which stimulates bone resorption [19] and is associated with substantial bone loss continuing into old age [20]. The mean age at menopause is around 51 years [21]. However, approximately $1 \%$ of women develop premature ovarian failure (POF) and go through an early menopause by the age of 40 [22]. Although POF patients commonly receive hormone replacement therapy (HRT), their BMD remains significantly lower than that of age-matched controls. POF thus remains one of the major risk factors for osteoporosis in women.

With such a variety of risk factors for a reduced BMD and osteoporosis, there is a need to identify patients who are at increased risk of sustaining fractures [23]. The Royal College of Physicians in the United Kingdom have issued guidelines for referring patients for bone densitometry investigations based on clinical risk factors [8]. Similar guidelines have also been published by the European Foundation of Osteoporosis and Bone Disease [3], the National Osteoporosis Foundation [24] and the International Society for Clinical Densitometry [25]. All of the aforementioned guidelines include early menopause as a recognised risk factor for osteoporosis and as a basis to refer patients for a bone density examination.

The most widely accepted method of performing BMD scans to establish a diagnosis of osteoporosis is the DXA technique. DXA involves scanning the lumbar spine and hip, measurement sites chosen because they are the most prone to osteoporotic fractures. However, DXA scanners are relatively expensive pieces of equipment and their availability is generally restricted to major hospitals. If the diagnostic benefits of bone densitometry are to be fully realised, smaller, cheaper devices are required. One possibility is the introduction of small DXA scanners designed to scan only the forearm. This technique is referred to as peripheral DXA (pDXA). Another peripheral technique is quantitative ultrasound (QUS). Measurements of broadband ultrasonic attenuation (BUA) in the calcaneus can discriminate elderly women with hip fractures and there is a consensus that QUS has a potentially valuable role to assess fracture risk. The attraction to QUS devices is that as well as being cheap and portable, they do not use ionising radiation.

Since fractures may be present or absent in patients with similar BMD, bone strength cannot depend exclusively on bone density, but also on bone architecture [26]. Use of ultrasound in fracture risk assessment is thus advantageous as it seems to provide structural information, e.g. data on trabecular orientation [27], and can also reflect the mechanical properties of bone [28] in addition to estimation of its density. The calcaneus is an easily accessible, weightbearing site, rich in trabecular bone and has been the most extensively studied site with QUS devices [29]. QUS measurements at this site have shown the ability to detect changes associated with age and menopause $[26,30]$. Measurements have also demonstrated the ability to differentiate healthy subjects from those with fractures [30,31] and also identifying those who are at an increased risk of fracture [32,33]. However, calcaneal QUS measurements can be unreliable in patients with ankle oedema. Variations in temperature (both ambient and of the patient's limb) are also believed to have an adverse effect on measurements [34].

An alternative, non-weight-bearing site for the assessment of bone mass using QUS is at the phalanges. The phalanges are composed of predominantly cortical bone and the regions of interest are easily accessible. The DBM Sonic Bone Profiler (IGEA, Italy) has been designed to transmit a single ultrasound burst through the distal metaphyses of the proximal phalanges. Bone resorption results in enlargement of the medullary canal and other structural changes, decreasing the ultrasound velocity and altering the characteristics of the signal arriving at the receiver probe [35]. The hand is very sensitive to these changes and is thus ideal for such assessment [36]. Previous studies using the DBM Sonic device have reported good precision with a coefficient of variation of $0.34 \%$ [37]. There is also evidence suggesting that phalangeal QUS measurements may be more sensitive than calcaneal measurements in identifying trends due to ageing and menopause [38].

The purpose of the present study was to examine the correlation between QUS parameters as measured at the proximal phalanges using the DBM Sonic Bone Profiler and conventional DXA measurements of BMD at the spine and hip in Caucasian women with POF. The study 
population was readily available to us from a local menopause clinic and hence women with POF were chosen as a specific group in which to assess the correlation. It was not the aim of the present study to determine the fracture discrimination capability of the DBM Sonic device.

\section{METHODS}

\section{Study Population}

Thirteen Caucasian women (mean age 35.7 yrs, range 30.5 - 40.6 yrs), suffering from POF, were recruited from the menopause clinic at Chelsea and Westminster Hospital (London, UK). The control group consisted of 19 age-matched healthy Caucasian women (mean age 32.9 yrs, range $20.4-40.1$ yrs) and were recruited with advertisements placed around the hospital sites. The study was approved by the Charing Cross Hospital Research Ethics Committee and all participants gave written informed consent.

Health assessment was carried out using a detailed questionnaire which included questions relating to known risk factors for osteoporosis, detailed medical history of POF, and use of HRT. Women on HRT were not excluded from the study. However, those with known risk factors for osteoporosis were excluded. These included women with a previous atraumatic fracture, and those on therapies or with conditions known to affect BMD (including thyroid conditions, malabsorption and use of corticosteroids).

\section{Dual X-ray Absorptiometery}

BMD was measured with a Lunar Prodigy (GE Healthcare, Madison, WI) DXA scanner. Measurements were performed at the lumbar spine (L1-L4), hip (total and neck of femur). In addition to recording BMD at these sites, we also recorded the T-score (a measure of how a subjects BMD value compares to those of a normal young adult at PBM - defined in terms of the standard deviation of young normal subjects) and Zscore (a measure of how a subjects BMD compares to an age-matched population - defined in terms of the standard deviation of the age-matched population). BMD measurement of the spine could not be obtained in one subject due to a navel ring, which could not be removed.

\section{QUS}

QUS measurements were performed on all subjects using the DBM Sonic Bone Profiler (IGEA, Carpi, Italy). A calliper was used to position the two probes (transmitter and receiver) laterally on either side of the metaphysis of the proximal phalanx, with ultrasound gel to achieve coupling. An ultrasound signal of $1.2 \mathrm{MHz}$ frequency is transmitted through the finger and an amplitude-dependent speed of sound (AD-SoS) parameter is generated, which depends on both the amplitude and the velocity of the signal received. Finger thickness and AD-SoS measurements were obtained for digits II-V of the non-dominant hand. Mean AD-SoS across all four digits was also obtained. Further measurement parameters included the Ultrasound Bone Profile Index (UBPI), a number between 0 and 1 describing the fracture risk [51], and the Bone Transmission Time (BTT) - the interval between the first received signal and the received signal that is propagated through soft tissue only [39]. T-scores and $\mathrm{Z}$-scores based on the AD-SoS values are also automatically generated.

Duplicate measurements were performed for each subject to estimate precision, which was expressed as the coefficient of variation $(\mathrm{CV})$. The mean values of the two measurements for each parameter were recorded and used for analysis. Ambient room temperature was kept constant with an air conditioning system as previous reports have suggested that QUS measurements are temperature-dependent [40]. Daily quality control measurements to ensure consistency of calibration were performed with a phantom supplied by the manufacturer.

\section{Statistical Analysis}

Data analysis was performed using Microsoft Excel (Seattle, WA, USA). Student's t-test was used to compare differences in demographic variables and in QUS and DXA parameters between the two groups. Pvalues of less than 0.05 were considered to be statistically significant. Linear regression analysis was carried out to determine the correlation between QUS and DXA parameters in (i) patients only ( $\mathrm{n}=13$ ), (ii) control group only $(\mathrm{n}=19)$ (iii) all participating subjects $(n=32)$. The following comparisons were made: Spine BMD v AD-SoS; Spine BMD v BTT; Spine BMD v QUS Z-score; Spine Z-score v AD-SoS; Spine Z-score v BTT; Spine Z-score v QUS Z-score. The same correlations were also determined for hip BMD and hip Z-scores. The correlation of QUS and DXA parameters with weight and BMI was also examined.

\section{RESULTS}

Duplicate QUS measurements with repositioning on all 32 patients were combined to give short-term precision (CV\%) of $0.37 \%, 3.36 \% 1.67 \%$ for $\mathrm{AD}-\mathrm{SoS}$, UBPI and BTT respectively.

Table 1 gives a summary of the demographic data, BMD parameters and QUS parameters for the patient group and the controls. Student's t-test showed no significant differences in any of the demographic or measurement parameters between the two groups. 
Linear regression analysis was used to establish the correlation between DXA and QUS parameters. None of the correlations between UBPI and the DXA parameters were significant. The correlation between remaining QUS parameters and spine BMD are shown in Table 2. A significant correlation $(\mathrm{r}=0.66)$ was observed in POF patients between spine BMD and BTT. This relationship remained significant $(\mathrm{r}=0.48)$ when all 32 subjects were included in the analysis (Figure 1). All QUS variables (AD-SoS, BTT and Z-score) showed significant correlations ( $\mathrm{r}=0.47$ to 0.60 ) with spine $\mathrm{Z}$ scores (Table 2, Figures 2-4). The T-score is a linear function of AD-SoS (for QUS measurements) and BMD for (DXA measurements). T-score correlations therefore provide no additional information and were excluded from the analysis. QUS correlations with DXA measurements at the hip (total hip and femoral neck sites) were not significant (Table 2). Normalising the QUS parameters by age and BMI did not improve the strength of correlation between any of above

\begin{tabular}{|l|c|c|c|}
\hline & $\begin{array}{c}\text { All women } \\
\mathrm{n}=32\end{array}$ & $\begin{array}{c}\text { POF group } \\
\mathrm{n}=13\end{array}$ & $\begin{array}{c}\text { Control group } \\
\mathrm{n}=19\end{array}$ \\
\hline Age (years) & $34.1 \pm 5.1$ & $35.7 \pm 3.4$ & $32.9 \pm 5.8$ \\
Height (m) & $1.66 \pm 0.06$ & $1.65 \pm 0.08$ & $1.67 \pm 0.05$ \\
Weight (kg) & $68.3 \pm 12.3$ & $68.5 \pm 15.7$ & $68.3 \pm 9.9$ \\
BMI (kg/m2) & $24.8 \pm 4.6$ & $25.2 \pm 5.9$ & $24.5 \pm 3.7$ \\
\hline QUS DBM Sonic & & & \\
AD-SoS (m/s) & $2128 \pm 74$ & $2138 \pm 85$ & $2121 \pm 67$ \\
UBPI & $0.72 \pm 0.14$ & $0.74 \pm 0.14$ & $0.71 \pm 0.14$ \\
BTT ( $\mu \mathrm{s})$ & $1.54 \pm 0.21$ & $1.53 \pm 0.21$ & $1.54 \pm 0.22$ \\
\hline DXA Lunar & & & \\
Spine BMD (g/cm 2$)$ & $1.19 \pm 0.11$ & $1.19 \pm 0.11$ & $1.18 \pm 0.11$ \\
Hip (total) BMD (g/cm $)$ & $1.00 \pm 0.12$ & $0.99 \pm 0.11$ & $1.00 \pm 0.13$ \\
Hip (neck) BMD $\left(\mathrm{g} / \mathrm{cm}^{2}\right)$ & $1.03 \pm 0.13$ & $1.02 \pm 0.10$ & $1.04 \pm 0.15$ \\
\hline
\end{tabular}

Table 1: Demographic, QUS and DXA data for patient group, control group and all subjects (mean and standard deviation). Student's t-tests show $\mathrm{p}>0.1$ for all parameters

\begin{tabular}{|l|l|c|c|c|}
\hline & & $\begin{array}{c}\text { AD-SoS } \\
(\mathrm{m} / \mathrm{s})\end{array}$ & BTT $(\mu \mathrm{s})$ & Z-score \\
\hline $\begin{array}{l}\text { Spine BMD } \\
(\mathrm{g} / \mathrm{cm} 2)\end{array}$ & POF group & 0.12 & $0.66^{*}$ & 0.12 \\
& Control group & 0.34 & 0.37 & 0.34 \\
& Pooled data & 0.24 & $0.48^{*}$ & 0.24 \\
\hline Spine Z-scores & POF group & 0.43 & 0.54 & 0.43 \\
& Control group & $0.60^{*}$ & 0.43 & $0.59^{*}$ \\
& Pooled data & $0.51^{*}$ & $0.47^{*}$ & $0.51^{*}$ \\
\hline $\begin{array}{l}\text { Total Hip BMD } \\
\text { g/cm2) }\end{array}$ & POF group & 0.08 & 0.52 & 0.08 \\
& Control group & 0.001 & 0.09 & 0.009 \\
& Pooled data & 0.04 & 0.22 & 0.03 \\
\hline $\begin{array}{l}\text { Total Hip } \\
\text { Z-scores }\end{array}$ & POF group & 0.15 & 0.49 & 0.15 \\
& Control group & 0.12 & 0.12 & 0.13 \\
& Pooled data & 0.12 & 0.23 & 0.12 \\
\hline
\end{tabular}

Table 2: Correlation (r-values) between three QUS parameters and spine BMD shown for all subjects $(n=32)$, POF group $(n=13)$ and control group $(\mathrm{n}=18)$. Results are also shown for the correlation with Total Hip BMD and Z-score.*Statistically Significant $(\mathrm{p}<0.05)$ comparisons.

As well as providing the mean AD-SoS values, the Bone Profiler displays data for the thickness and ADSoS for each of the four phalanges measured. Correlations between the AD-SoS values for individual fingers and the DXA parameters were again moderate ( $\mathrm{r}$ $=0.44-0.63)$ but only significant when compared to spine Z-scores (Table 3).

Further analysis was carried out to compare QUS parameters with weight and BMI. Our results show a

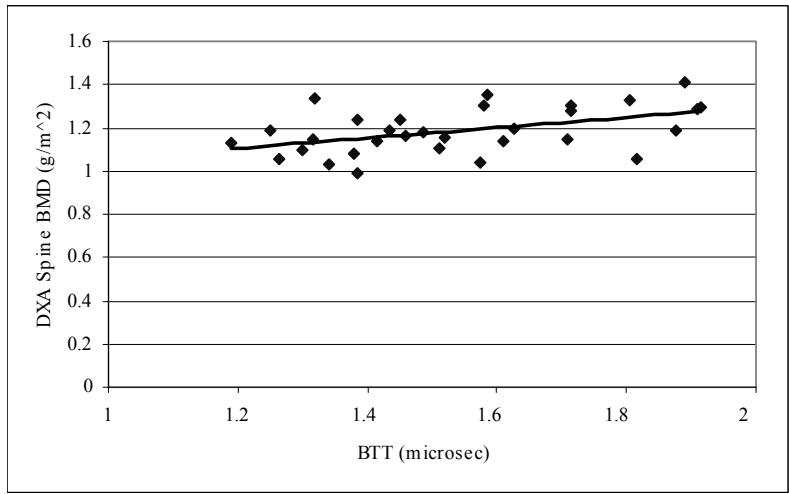

Figure 1: Graph showing the positive correlation between the QUS parameter BTT, and spine BMD for all subjects $(\mathrm{n}=31 ; \mathrm{r}=0.48 ; \mathrm{p}=$ $0.007)$

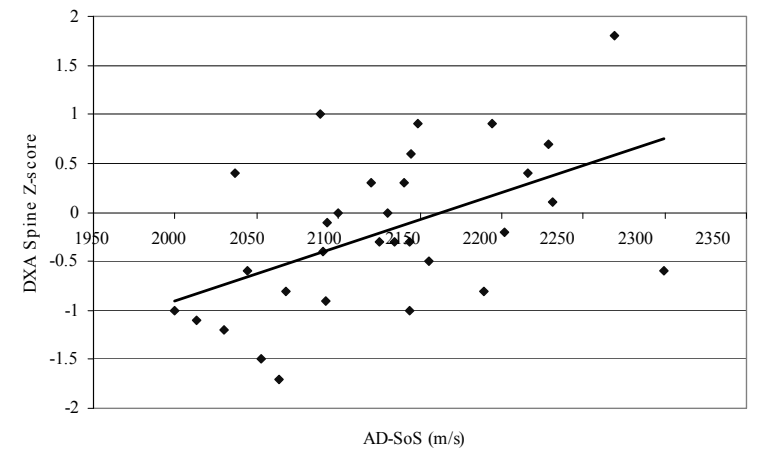

Figure 2: Graph showing the positive correlation between the QUS parameter $\mathrm{AD}-\mathrm{SoS}$, and spine Z-score for all subjects $(\mathrm{n}=31 ; \mathrm{r}=0.51$; $\mathrm{p}=0.003)$.

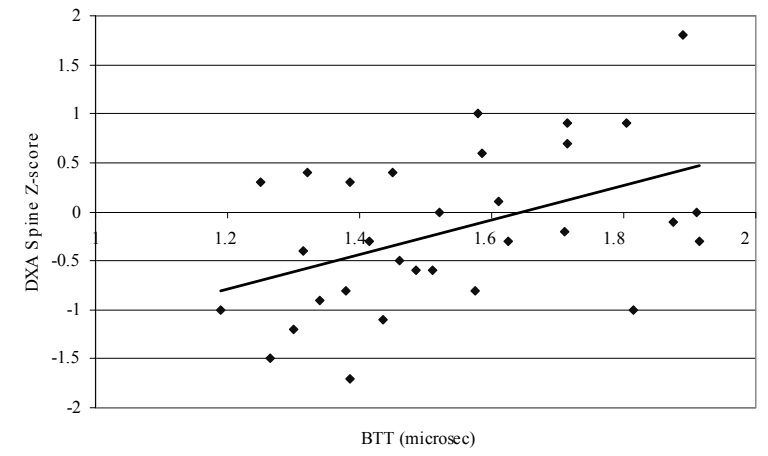

Figure 3: Graph showing the positive correlation between the QUS parameter BTT, and spine Z-score for all subjects $(\mathrm{n}=31 ; \mathrm{r}=0.47 ; \mathrm{p}=$ $0.008)$ 


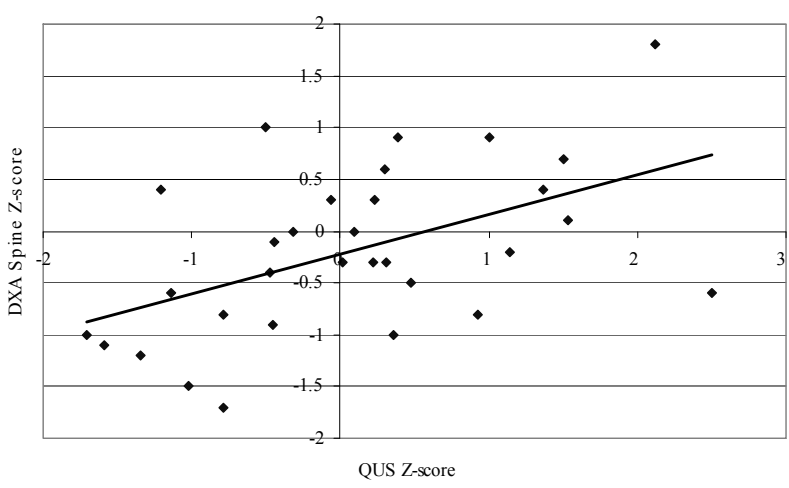

Figure 4: Graph showing the positive correlation between the QUS $\mathrm{Z}$-score and spine $\mathrm{Z}$-score for all subjects $(\mathrm{n}=31 ; \mathrm{r}=0.51 ; \mathrm{p}=0.003)$.

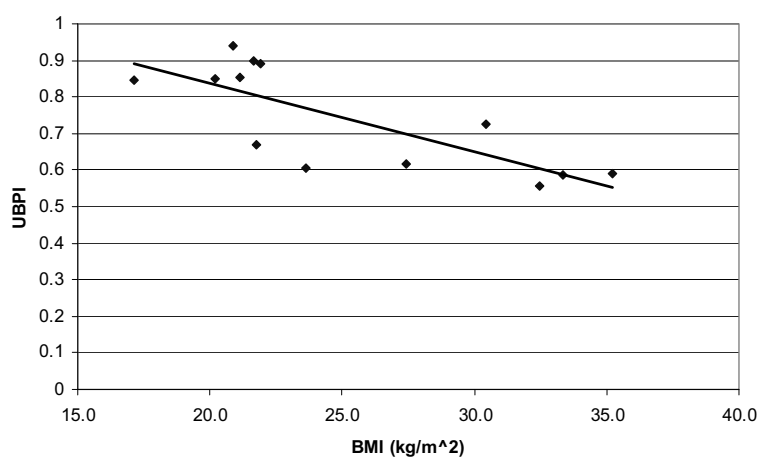

Figure 5: Graph showing the inverse correlation between BMI and the QUS parameter UBPI for the POF group $(n=13 ; r=-0.78 ; p=$ 0.002 )

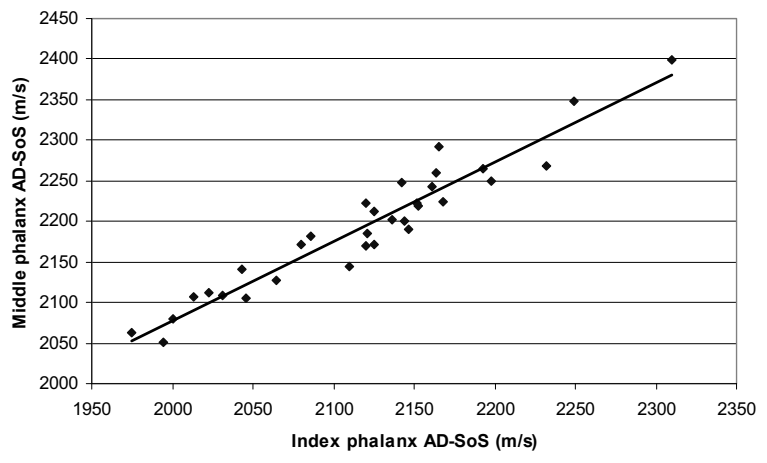

Figure 6: Graph showing the positive correlation between AD-SoS measurements obtained from the index (phalanx II) and the middle (phalanx III) fingers, for all subjects $(n=32 ; r=-0.96 ; p<0.0001)$.

Table 3: Correlation (r-values) between individual phalanx AD-SoS values and the spine $Z$-score measurements from the DXA for all subjects $(n=31)$, POF group $(n=13)$ and the control group $(n=18)$

*Statistically Significant $(\mathrm{p}<0.05)$

\begin{tabular}{|l|l|c|c|c|c|}
\hline & & $\begin{array}{c}\text { Phalanx II } \\
\text { AD-SoS }(\mathrm{m} / \mathrm{s})\end{array}$ & $\begin{array}{c}\text { Phalanx III } \\
\text { AD-SoS }(\mathrm{m} / \mathrm{s})\end{array}$ & $\begin{array}{c}\text { Phalanx IV } \\
\text { AD-SoS }(\mathrm{m} / \mathrm{s})\end{array}$ & $\begin{array}{c}\text { Phalanx V } \\
\text { AD-SoS (m/s) }\end{array}$ \\
\hline Spine & POF group & 0.38 & 0.30 & 0.53 & 0.44 \\
Z-score & Control group & $0.59 *$ & $0.63^{*}$ & 0.39 & $0.50^{*}$ \\
& Pooled data & $0.49 *$ & $0.47^{*}$ & $0.44^{*}$ & $0.47 *$ \\
\hline
\end{tabular}

\section{DISCUSSION} *Statistically Significant $(\mathrm{p}<0.05)$

reasonably strong inverse correlation between all 3 QUS parameters (AD-SoS, UBPI and BTT) and BMI (Table 4). The strongest correlation was with UBPI in the POF patient group $(\mathrm{r}=-0.78)$ (Figure 5). Similar inverse correlations were observed $(r=-0.48$ to -0.76$)$ between QUS parameters and subjects' weight. Similar values were also observed when comparing weight and $\mathrm{BMI}$ with $\mathrm{AD}-\mathrm{SoS}$ values from individual fingers. The correlation between height and BMI was also established for DXA measurements at the spine and hip. Moderate but significant positive correlations were observed with spine and hip BMD $(r=0.37-0.62)$.

Finally, the correlation of AD-SoS values between the digits was established and found to be highly significant $(\mathrm{p}<0.0001)$. The strongest correlation $(\mathrm{r}=0.96)$ was observed between the index and middle fingers (Figure 6). Student's t-test demonstrated significant differences in AD-SoS and thickness values between fingers. These observations were similar in the patient group and in the controls. The AD-SoS values were greatest in phalanx III, followed by IV, II and V $(\mathrm{p}<0.02)$.

Because of the growing demand for bone densitometry services, there is a need for cheap, safe and portable devices that can be used in a primary care setting to assess BMD and fracture risk. The ability of a device to independently assess fracture risk is best established through prospective fracture studies which can be expensive and time consuming. When evaluating new bone density scanners it is of interest to examine the correlation with DXA measurements at the spine and hip - the established 'gold standard' bone density measurements. In this study, we investigated the correlation between QUS parameters obtained using the DBM Sonic Bone Profiler and spine and hip

Table 4: Correlation ( $\mathrm{r}$ values) between three QUS parameters and BMI; split by POF group $(n=13)$, control group $(n=19)$ and the pooled data $(\mathrm{n}=32)$. Correlations are also shown for weight.

\begin{tabular}{|l|l|c|c|c|}
\hline & & $\begin{array}{c}\text { AD-SoS } \\
(\mathrm{m} / \mathrm{s})\end{array}$ & Z-score & UBPI \\
\hline BMI \\
$(\mathrm{kg} / \mathrm{m} 2)$ & POF group & $-0.69^{*}$ & $-0.69^{*}$ & $-0.78^{*}$ \\
& Control group & $-0.48^{*}$ & $-0.48^{*}$ & -0.25 \\
& Pooled data & $-0.58^{*}$ & $-0.58^{*}$ & $-0.49^{*}$ \\
\hline Weight & POF group & -0.49 & -0.50 & $-0.76^{*}$ \\
& Control group & $-0.48^{*}$ & $-0.48^{*}$ & -0.25 \\
& Pooled data & $-0.48^{*}$ & $-0.48^{*}$ & $-0.57^{*}$ \\
\hline
\end{tabular}


measurements acquired on a Lunar Prodigy system.

The precision analysis of our QUS measurements returned $\mathrm{CV} \%$ values of $0.37-3.36 \%$ for the various parameters. The AD-SoS precision compare favourably with previously reported results [41,42]. The precision for the Prodigy DXA device has been previously quoted as $1.0 \%$ for the spine (L1-L4), $0.9 \%$ for hip (total) and $1.5 \%$ for femoral neck [43].

Linear regression analysis between DXA and QUS parameters showed a significant correlation of 0.66 $(\mathrm{p}=0.01)$ between the BTT value and spine BMD in women with POF, although this was not the case for other QUS parameters. BTT is a measure of the time delay between the two signals arriving at the receiver probe, with one having passed through bone and the other through the soft tissue phase. The significant correlation observed in our study could indicate that an improved bone structure at the phalanx correlates with higher bone density at the spine, and thus allows a more rapid transmission of the ultrasound signal through bone compared to soft tissue. Although much of the literature currently focuses on the AD-SoS values provided by the QUS device, Montagnani et al [44] have presented data highlighting the importance of BTT as the parameter most comparable to DXA and with the greatest ability to predict osteoporotic fractures.

Significant correlations of $r=0.5$ to 0.6 were observed between spine Z-scores and AD-SoS, as well as with BTT. QUS and DXA Z-scores showed a moderate correlation when the data from patients and controls was pooled $(\mathrm{r}=0.51)$. Although $\mathrm{Z}$-scores are not used in the diagnosis of osteoporosis our results suggest that this relationship between $Z$-scores merits further investigation. If QUS Z-scores can predict DXA Zscores, it may suggest a role for QUS measurements as a screening tool. It has previously been suggested that phalangeal QUS Z-scores may be a useful tool in the screening of bone disturbances in young patients with type I diabetes mellitus [45]. It is possible that phalangeal QUS may also have a role in other disease states affecting bone.

Regression analysis demonstrated that AD-SoS, UBPI and QUS Z-score parameters are all inversely related with both BMI and weight. Although the Bone Profiler accounts for soft tissue content, weak but significant correlations ( $r=-0.30$ to -0.32 ) between AD-SoS and BMI have been reported previously $[41,46]$. Interestingly, BTT does not show a similar correlation but was the only QUS parameter to show a positive correlation with height in the POF group. A similarly significant correlation was seen between spine BMD and height $(\mathrm{r}=0.56)$, which strengthens the argument for further research into the potential value of BTT as a predictor of spine BMD. Currently however, the association between QUS and BMI remains unclear with some showing evidence of a significant correlation [47,48], whilst other data remains inconclusive [49]. Alenfeld et al. [48] postulate QUS may depend on body weight and BMI as the soft tissue surrounding the phalanges influences both acoustical contact and velocity.

Repeated student's t-test analyses for the differences in mean thickness between phalanges II to V showed a significant, progressive increase in width from index to small fingers. However, the mean AD-SoS values were significantly greater in the middle finger, followed by the ring, index and small fingers, implying that the QUS AD-SoS parameter is indeed not simply a function of the distance between the probes, but can identify quantitative differences between the structures of the digits. The potential of QUS measurement to reflect bone structure, as well as bone density, has in fact been one of the driving forces for QUS densitometry. QUS measurements may have a role in assessing fracture risk in patients on corticosteroids for example [15], which are known to affect the structure of trabecular bone and have a detrimental effect 'over and beyond' that reflected in a DXA measurement of low bone mass alone [50].

Analysis of the collected data has shown significant correlations between a number of QUS and DXA device parameters, as well as upon comparison with demographics. Perhaps the most promising of these is the correlation of spinal BMD with BTT and, with an r2 value of 0.44 in the POF group, offers the best predictive ability of BMD compared to the other QUS parameters.

However, other studies have highlighted the use of alternative QUS parameters for the estimation of BMD. Alexandersen et al [26] demonstrated weak but significant correlations between AD-SoS and BMD at the spine and hip (femoral neck) $(\mathrm{r}=0.21$ for both sites), whereas Wuster et al [51] obtained higher correlations $(\mathrm{r}=0.46$ and $\mathrm{r}=0.36$ spine and hip BMD, respectively). The lack of concordance with our study may lie in the differences between populations from Denmark, Scandinavia and the UK, respectively. Furthermore, the Bone Profiler is a device with a higher sensitivity to skeletal changes occurring in the early postmenopausal period (in the range 50-65), which encompasses the population used by Wuster et al [51] and thus providing the strongest correlations. The mean age in the Alexandersen study [26] was 69.9 years, at which age postmenopausal changes can still be identified. However, our study had a mean age for all subjects of 34.1 years, with one group having been through an early menopause and the other group acting as controls. Thus, differences in the population age and 
inconsistencies of menopausal state of the subjects may account for the discrepancies. Other studies have also shown negative associations of QUS parameters with age [52]. However, the unique patient group we selected has not been assessed by others in this manner. This adds a confounding factor to our data making comparison difficult. An unlikely alternative explanation for the discrepancies between studies is user-dependence which was highlighted as significant factor affecting the accuracy of measurements in a study by Krieg et al [53] who carried out measurements using the DBM Sonic on over 7000 women.

One advantage of assessing the human phalanges is their sensitivity to early changes in bone mass [54], and work originally based on animal studies has supported the argument that phalangeal ultrasound can identify architectural characteristics of bone [35]. However, unlike the calcaneus, phalangeal bone is not subject to weight-bearing activity and thus provides weaker correlations compared to peripheral weight-bearing sites [54]. Furthermore, inaccurate positioning of the calliper on the phalanx can lead to errors, as the joint itself has greater trabecular bone content than the metaphysis [55] and can therefore contribute to operator errors. QUS values are also dependent on the temperature of the limb, which can be difficult to control in variable climatic conditions. However, in the present study room temperature was controlled using an air conditioning system. One further source of measurement error that has been reported is that the phalangeal joints are common sites for osteoarthritis [26], which could interfere with QUS measurements.

The stronger correlation of our results occurring between QUS and DXA at the spine, as opposed to at the hip, may partly be explained by the fact that trabecular rich vertebrae are more sensitive in identifying changes occurring with age or therapy compared to the hip.

As well as the relatively small sample size, there were further limitations to our study. HRT is known to significantly reduce the impact of the menopause on bone loss [56] but its duration of use in the present study (4 months to $7 \mathrm{yrs}$ ) varied widely between patients. Uygur et al [22] have suggested that current HRT regimes, designed for women undergoing natural menopause, may be inappropriate for some women with POF and thus cause further variations in the effects of HRT in POF groups. The use of HRT may have different effects on QUS and DXA parameters.

The potential benefits for phalangeal QUS to be adopted as a screening device could extend beyond its correlation with DXA. Other studies have shown the ability of the phalangeal QUS device to identify changes associated with age and with the menopause, but also to predict fracture risk [56-59]. Its relatively low $\mathrm{CV} \%$ values can also allow frequent monitoring of bone health. Although QUS cannot provide a direct measurement of BMD itself, its potential for detecting structural changes may be more beneficial than established areal BMD measurements.

Our study suggests that there may be a need to move away from focusing on the AD-SoS parameter in place of the BTT, and even a greater role for Z-scores. BTT has shown stronger correlation with DXA values and stronger predictive $\mathrm{r} 2$ ability compared to other QUS parameters. Whilst some authors have described a combination of clinical risk factors and QUS values in the form of a 'nomogram' as the ideal method of screening for increased fracture risk [60], others believe the complex soft tissue-bone-ultrasound interactions and the resultant waveform they produce should be analysed [51]. In either case, the aim is to identify patients at risk of fracture before the fracture occurs. Possible further roles for phalangeal QUS may include measurements in children on long-term corticosteroids. All this is only possible through studies such as this being able to identify and harness the great expectations with which these devices were introduced, and allow early preventative interventions to be taken in high-risk groups.

\section{CONCLUSION}

We have reported moderate but significant correlations between phalangeal QUS and DXA parameters. The strongest correlation was observed between BTT and spine BMD, as well as between the Zscores from the two devices. QUS parameters also demonstrated an inverse correlation with weight and BMI. These correlations were seen within the POF group, control group and when data for all patients was pooled. Our data support further studies to evaluate the ability of phalangeal QUS measurements to independently assess fracture risk in patients with and without different risk factors. Such studies will help establish whether phalangeal QUS can be used as a screening tool in the primary care setting. Currently, the best measurement sites for QUS and the ideal parameters with which to form guidelines still have to be established.

\section{ACKNOWLEDGMENTS}

We would like to thank Dr Nick Panay at the Chelsea and Westminster hospital (London UK) for help with recruitment of patients with POF.

\section{REFERENCES}

1. Cooper C, Campion G, Melton LJ Hip fractures in the elderly; a 
world-wide projection. Osteoporosis Int 1992; 2:285-289.

2. Ray NF, Chan JK, Thamer M, Melton LJ Medical expenditures for the treatment of osteoporotic fractures in the United States in 1995: report from the National Osteoporosis Foundation. J Bone Miner Res 1997; 12:24-35

3. Kanis JA, Delmas P, Burckhardt P et al. on behalf of the European Foundation for Osteoporosis and Bone Disease. Guidelines for diagnosis and treatment of osteoporosis. Osteoporosis Int 1997; 7:390-406.

4. Black DM, Thompson DE, Bauer DC, et al. Fracture risk reduction with alendronate in women with osteoporosis: the fracture intervention trial. J Clin Endocrinol Metab 2000; $85: 4118-4124$

5. McClung MR, Geusens P, Miller PD, et al. Effect of risedronate on the risk of hip fracture in elderly women. N Engl J Med 2001; 344:333-340.

6. Neer RM, Arnaud CD, Zanchetta JR, et al. Effect of recombinant human parathyroid hormone (1-34) fragment on spine and non-spine fractures and bone mineral density in postmenopausal osteoporosis. N Engl J Med 2001; 344:14341441

7. Delmas PD, Ensrud KE, Adachi JD, et al. Efficacy of raloxifene on vertebral fracture risk in postmenopausal women with osteoporosis: four-year results from a randomized clinical trial. J Clin Endocrinol Metab 2001; 87:3609-3617.

8. Royal College of Physicians Osteoporosis: clinical guidelines for prevention and treatment. Update on pharmacological interventions and an algorithm for management. RCP 2000, London.

9. US Preventive Services Task Force. Screening for osteoporosis in postmenopausal women: recommendations and rationale. Arch Intern Med 2002; 137:526-528.

10. Bonjour J-P and Rizzoli R. Bone acquisition in adolescence. In Marcus R, Feldman D, Kelsey J (eds). Osteoporosis 1996; pp465-476. Academic Press, San Diego.

11. Kanis JA, Gluer C-C. An update on the diagnosis and assessment of osteoporosis with densitometry. Osteoporosis International. 2000; 11: pp. 192-202.

12. Cashman KD. Calcium intake, calcium bioavailability and bone health. British Journal of Nutrition. 2002; 87 (Supplement S2): pp. S169-S177.

13. Rideout CA, McKay HA, Barr SI. Self-reported lifetime physical activity and areal bone mineral density in healthy postmenopausal women: the importance of teenage activity. Calcified Tissue International. 2006; 79 (4): pp. 214-222.

14. Pons F, Peris P, Guanabens $\mathrm{N}$ et al. The effect of systemic lupus erythematosus and long-term steroid therapy on bone mass in pre-menopausal women. British Journal of Rheumatology. 1995; 34: pp. 742-6.

15. Chappard D, Josselin N, Rouge-Maillart C, et al. Bone microarchitecture in males with corticosteroid-induced osteoporosis. Osteoporosis International. 2007; 18 (4): pp. 487494.

16. Bainbridge PR, Eastell R. Indications for bone densitometry: do they identify patients with low bone mineral density? Current research in osteoporosis and bone mineral management. British Journal of Radiology. 1998; 5: pp. 5-6.

17. Seriolo B, Paolino S, Sulli A, et al. Bone metabolism changes during anti-TNF-alpha therapy in patients with active rheumatoid arthritis. Annals of the New York Academy of Sciences. 2006; 1069 (1): pp. 420-7.

18. Aitken JM, Hart DM, Anderson JB, et al. Osteoporosis after oophorectomy for non-malignant disease in premenopausal women. British Medical Journal. 1973; 2: pp. 325-8.

19. Genant HK, Cooper $\mathrm{C}$, Poor $\mathrm{G}$ et al. Interim report and recommendations of the World Health Organisation task-force for osteoporosis. Osteoporosis International. 1999; 10: pp. 259264.

20. Ensrud KE, Palermo L, Black DM, et al. Hip and calcaneal bone loss increase with advancing age: longitudinal results from the study of osteoporotic fractures. Journal of Bone and Mineral Research. 1995; 10: pp. 1778-87.

21. Kazerooni T, Talei AR, Sadeghi-Hassanabadi A, et al. Reproductive behaviour in women in Shiraz, Islamic Republic of Iran. Eastern Mediterranean Health Journal. 2000; 6 (2): pp. 517-521.

22. Uygur D, Sengul O, Bayar D, et al. Bone loss in young women with premature ovarian failure. Archives of Gynecology and Obstetrics. 2005; 273: pp. 17-19.

23. Johnell O, Kanis JA, Oden A, et al. Predictive value of BMD for hip and other fractures. Journal of Bone and Mineral Research. 2005; 20: pp. 1185-94.

24. National Osteoporosis Foundation Risk assessment. In: Physician's Guide to Prevention and Treatment of Osteoporosis. National Osteoporosis Foundation 1998; Washington DC, USA.

25. International Society for Clinical Densitometry. Official Positions and Official Pediatric Positions of the ISCD 2007.

26. Alexandersen P, de Terlizzi F, Tanko LB, et al. Comparison of quantitative ultrasound of the phalanges with conventional bone densitometry in healthy postmenopausal women. Osteoporosis International. 2005; 16: pp. 1071-78.

27. Gluer $\mathrm{CC}, \mathrm{Wu} \mathrm{CY}$, Genant HK. Broadband ultrasound attenuation signals depend on trabecular orientation: an in vitro study. Osteoporosis International. 1993; 3: pp. 185-191.

28. Bouxsein ML, Radloff SE. Quantitative ultrasound of the calcaneus reflects the mechanical properties of calcaneal trabecular bone. Journal of Bone and Mineral Research. 1997; 12: pp. 839-46.

29. Frost ML, Blake GM, Fogelman I. Does quantitative ultrasound imaging enhance precision and discrimination? Osteoporosis International. 2000; 11: pp. 425-433.

30. Frost ML, Blake GM, Fogelman I. Contact quantitative ultrasound: an evaluation of precision, fracture discrimination, age-related bone loss and applicability of the WHO criteria. Osteoporosis Interantional. 1999; 10: pp. 441-449.

31. He YQ, Fan B, Hans D, et al. Assessment of a new quantitative ultrasound calcaneus measurement: precision and discrimination of hip fractures in elderly women compared with dual X-ray absorptiometry. Osteoporosis International. 2000; 11 (4): pp. 354-360.

32. Bauer DC, Gluer CC, Cauley JA, et al. Broadband ultrasound attenuation predicts fractures strongly and independently of densitometry in older women. Archives of Internal Medicine. 1997; 157: pp. 629-634.

33. Woodhouse A, Black DM. BMD at various sites for the prediction of hip fractures: a meta analysis. Journal of Bone and Mineral Research. 2000; 15: pp. 1-145.

34. Johansen A, Stone MD. The effect of ankle oedema on bone ultrasound assessment at the heel. Osteoporosis International. 1997; 7: pp. 44-47.

35. Cadossi R, Cane V. Pathways of transmission of ultrasound energy through the distal metaphysis of the second phalanx of pigs. Osteoporosis International. 1996; 6: pp. 196-206.

36. Kleerekoper M, Nelson DA, Flynn MJ, et al. Comparison of radiographic absorptiometry with dual-X-ray absorptiometry and quantitative computed tomography in normal older white and black women. Journal of Bone and Mineral Research. 1994; 9: pp. 1745-9.

37. Di Stefano M and Isaia GV. Ability of ultrasound bone profile score(UBPS) to discriminate between fractured and not fractured osteoporotic women. Ultrasound in Medicine \& Biology 2002; 28(11-12), pp1485-1489. 
38. Pluskiewicz W, Drozdzowska B. Quantitative ultrasound (QUS) at the calcaneus and hand phalanges in Polish healthy postmenopausal women. Ultrasound in Medicine and Biology. 2001; 27 (3): pp. 373-7.

39. Guglielmi G, de Terlizzi F, Torrente I, et al. Quantitative ultrasound of the hand phalanges in a cohort of monozygotic twins: influence of genetic and environmental factors. Skeletal Radiology. 2005; 34 (11): pp. 727-735.

40. Pocock NA, Babichev A, Culton N, et al. Temperature dependency of quantitative ultrasound. Osteoporosis International. 2000; 11: pp. 316-320.

41. Ventura V, Mauloni M, Mura M, et al. Ultrasound velocity changes at the proximal phalanxes of the hand in pre-, peri- and postmenopausal women. Osteoporosis International. 1996; 6: pp. 368-375.

42. Joly J, Westhovens R, Borghs $\mathrm{H}$, et al. Reference curves and diagnostic sensitivity for a new ultrasound device for the phalanges, the DBMsonic 1200, in Belgian women. Osteoporosis International. 1999; 9 (4): pp. 284-9.

43. Shepherd J, Fan B, Lu Y, et al. Comparison of BMD precision for Prodigy and Delphi spine and femur scans. Osteoporosis International. 2006; 17 (9): pp. 1303-8.

44. Montagnani A, Gonnelli S, Cepollaro C, et al. Usefulness of bone quantitative ultrasound in management of osteoporosis in men. Journal of Clinical Densitometry. 2001; 4 (3): pp. 231-7.

45. Valerio G, del Puente A, Buono P, et al. Quantitative ultrasound of proximal phalanges in patients with type I diabetes mellitus. Diabetes Research and Clinical Practice. 2004; 64 (3): pp. 1616.

46. Halaba ZP, Konstantynowicz J, Pluskiewicz W, et al. Comparison of phalangeal ultrasound and dual energy X-ray absorptiometry in healthy male and female adolescents. Ultrasound in Medicine and Biology. 2005; 31 (12): pp. 161722.

47. Rico H, Aguado F, Arribas I, et al. Behaviour of phalangeal bone ultrasound in normal women with relation to gonadal status and body mass index. Osteoporosis International. 2001; 12: pp. 4505 .

48. Alenfeld FE, Wuster C, Funck C, et al. Ultrasound measurements at the proximal phalanges in healthy women and patients with hip fractures. Osteoporosis International. 1998; 8: pp. 393-8.

49. Gregg EW, Kriska AM, Salamone LM, et al. Correlates of quantitative ultrasound in the Women's Healthy Lifestyle Project. Osteoporosis International. 1999; 10: pp. 416-424.

50. Royal College of Physicians. Glucocorticoid-induced osteoporosis: guidelines for prevention and treatment. London: RCP; 2002.

51. Wuster C, Albanese C, De Aloysio D, et al. Phalangeal Osteosonogrammetry Study: age-related changes, diagnostic sensitivity, and discrimination power. Journal of Bone and Mineral Research. 2000; 15: pp. 1603-14.

52. Mele R, Masci G, Ventura V, et al. Three-year longitudinal study with quantitative ultrasound at the hand phalanx in a female population. Osteoporosis International. 1997; 7 (6): pp. 550-7.

53. Krieg MA, Cornuz J, Ruffieux C, et al. Prediction of hip fracture risk by quantitative ultrasound in more than 7000 Swiss women $\geq 70$ years of age: comparison of three technologically different bone ultrasound devices in the SEMOF study. Journal of Bone and Mineral Research. 2006; 21 (9): pp. 1457-63.

54. Rico H, Revilla M, Fraile E, et al. Metacarpal cortical thickness by computer radiography in osteoporosis. Bone. 1994; 15: pp. 303-6.

55. Schneider J, Bundschuh B, Spath C, et al. Discrimination of patients with and without vertebral fractures as measured by ultrasound and DXA osteodensitometry. Calcified Tissue
International. 2004; 74 (3): pp. 246-254.

56. Roben P, Barkmann R, Ullrich S, et al. Assessment of phalangeal bone loss in patients with rheumatoid arthritis by quantitative ultrasound. Annals of the rheumatic diseases. 2001; 60: pp. 670-7.

57. Pluskiewicz W, Drozdzowska B. Ultrasound measurements of proximal phalanges in Polish early postmenopausal women. Osteoporosis International. 1998; 8: pp. 578-583.

58. Hartl F, Tyndall A, Kraenzlin M, et al. Discriminatory ability of quantitative ultrasound parameters and bone mineral density in a population-based sample of postmenopausal women with vertebral fractures: result of the Basel Osteoporosis Study. Journal of Bone and Mineral Research. 2002; 17: pp. 321-330.

59. Guglielmi G, Cammisa M, De Serio A, et al. Phalangeal US velocity discriminates between normal and vertebrally fractured subjects. European Radiology. 1999; 9: pp. 1632-7.

60. Pluskiewicz W, Nowakowska J. Bone status after long-term anticonvulsant therapy in epileptic patients: evaluation using quantitative ultrasound of calcaneus and phalanges. Ultrasound Med Biol. 1997; 23: pp. 553-8.

61. Pongchaiyakul C, Panichkul S, Songpatanasilp T et al. A normogram for predicting osteoporosis risk based on age, weight and quantitative ultrasound measurement. Osteoporosis International. 2007; 18: pp. 525-531.

Tandip Mann (BSc in Surgery \& Anaesthesia) is a 5 th year medical student.

Alison McGregor $(\mathrm{PhD})$ is a reader in Biodynamics.

Rajesh Patel $(\mathrm{PhD})$ is the Head of Academic Bone densitometry and Clinical Studies. 